\title{
Evolving Systems: Adaptive Key Component Control and Inheritance of Passivity and Dissipativity
}

\author{
S.A.Frost ${ }^{\mathrm{a}, *}$, M.J.Balas ${ }^{\mathrm{b}}$ \\ ${ }^{a}$ Intelligent Systems Division, NASA Ames Research Center, Moffett Field, CA USA \\ ${ }^{b}$ Department of Electrical and Computer Engineering, University of Wyoming, Laramie, WY, USA
}

\begin{abstract}
We propose a new framework called Evolving Systems to describe the self-assembly, or autonomous assembly, of actively controlled dynamical subsystems into an Evolved System with a higher purpose. Autonomous assembly of large, complex flexible structures in space is a target application for Evolving Systems. A critical requirement for autonomous assembling structures is that they remain stable during and after assembly. The fundamental topic of inheritance of stability, dissipativity, and passivity in Evolving Systems is the primary focus of this research. In this paper, we develop an adaptive key component controller to restore stability in Nonlinear Evolving Systems that would otherwise fail to inherit the stability traits of their components. We provide sufficient conditions for the use of this novel control method and demonstrate its use on an illustrative example.
\end{abstract}

Keywords: Evolving Systems, stability, adaptive control, dissipativity, passivity

\section{Introduction}

We have proposed a new framework called Evolving Systems to describe the self-assembly, or autonomous assembly, of actively controlled dynamical subsystems into an Evolved System with a higher purpose [1,2]. The components of an Evolving System self-assemble, or mate, to complete the Evolved System. The Evolving Systems framework provides a scalable, modular architecture to model and analyze the subsystem components, their connections to other components, and the Evolved System. Ultimately, once all the components of an Evolving System have joined together to form the fully Evolved System, it will have a new, higher purpose that could not have been achieved by the individual components collectively.

Autonomous assembly of large, complex structures in space, or on-orbit assembly, is an excellent application area for Evolving Systems. Future space missions will require on-orbit assembly of large aperture (greater than 10 meters) space systems, possibly at distant locations that prohibit astronaut intervention [3]. Other applications for Evolving Systems can be found in $[2,4]$. These applications motivate the development of flexible structure Evolving Systems, which are mechanical dynamical systems consisting of actively controlled flexible structure components joined together by compliant forces.

\footnotetext{
*Corresponding author. Address: NASA Ames Research Center, M/S 269-3, Moffett Field, CA USA 94035

Email addresses: susan.a.frost@nasa.gov (S.A.Frost), mbalas@uwyo.edu (M.J.Balas) 
A critical requirement for autonomous self-assembling structures is that they remain stable during and after assembly. We say that a subsystem trait is inherited by an Evolving System when the system retains the properties of the trait after assembly. The inheritance of subsystem traits, such as controllability, observability, stability, passivity, and dissipativity, is an important research topic.

The fundamental topic of inheritance of stability, passivity, and dissipativity in Evolving Systems is our primary focus. In this paper, we develop an adaptive key component controller to restore stability to Nonlinear Evolving Systems that would otherwise fail to inherit the stability traits of their components. We provide sufficient conditions for the use of the control method. Sufficient conditions for the inheritance of passivity and dissipativity traits in Nonlinear Evolving Systems are also given. For linear time-invariant (LTI) systems, we show that strict dissipativity, strict passivity, and strict positive real are equivalent properties. Finally, we demonstrate the adaptive key component stability restoring controller on an illustrative example.

Decentralized control theory and analysis have been applied to the control of large interconnected systems; see the excellent survey paper by Nils Sandell [5] on this topic. Generally, decentralized control has been used to decrease the complexity of the control issues affecting large interconnected systems. A decentralized adaptive control approach presented in [6] differs from the approach developed here in many ways, including its use of the system's inputs and outputs. The ideas addressed by decentralized control theory are related, but not equivalent, to the Evolving Systems viewpoint.

On the experimental side, a research group at the Information Sciences Institute at the University of Southern California (USC) has been conducting research in self-reconfigurable, autonomous robots and systems. They have conducted experimental work to study the feasibility of techniques for assembling large space structures as part of their FIMER (Free-flying Intelligent MatchmakER robots) project [7,8]. This group uses a distributed control method with simple proportional derivative control laws for the self-assembly of components.

\section{Mathematical Formulation of Evolving Systems}

In this section we give the general mathematical formulation of nonlinear time-invariant Evolving Systems. See [2, 4] for a more detailed description of the Evolving Systems formulation. Often, applications have significant nonlinearities that must be accounted for in the system model. Hence, the framework presented here allows nonlinear dynamics in the system model.

The components are the building blocks of the Evolving System. Consider a system of $L$ individually actively controlled components, where the dynamical equations of the components are given by

$$
\left\{\begin{aligned}
\dot{\mathbf{x}}_{i} & =f_{i}\left(\mathbf{x}_{i}, \mathbf{u}_{i}\right) ; \mathbf{x}_{i}(0)=\mathbf{x}_{i 0} \\
\mathbf{y}_{i} & =g_{i}\left(\mathbf{x}_{i}, \mathbf{u}_{i}\right)
\end{aligned}\right.
$$

where $i=1,2, \ldots, L, \mathbf{x}_{i} \equiv\left[\begin{array}{llll}x_{1}^{i} & x_{2}^{i} & \cdots & x_{n_{i}}^{i}\end{array}\right]^{T}$ is the component state vector,

$\dot{\mathbf{x}}_{i} \equiv\left[\begin{array}{llll}\dot{x}_{1}^{i} & \dot{x}_{2}^{i} & \cdots & \dot{x}_{n_{i}}^{i}\end{array}\right]^{T}, \mathbf{u}_{i} \equiv\left[\begin{array}{llll}u_{1}^{i} & u_{2}^{i} & \cdots & u_{m_{i}}^{i}\end{array}\right]^{T}$ is the control input vector,

$\mathbf{y}_{i} \equiv\left[\begin{array}{llll}y_{1}^{i} & y_{2}^{i} & \cdots & y_{p_{i}}^{i}\end{array}\right]^{T}$ is the vector of sensed outputs, and $\mathbf{x}_{i_{0}} \equiv\left[\begin{array}{llll}x_{1_{0}}^{i} & x_{2_{0}}^{i} & \cdots & x_{n_{0}}^{i}\end{array}\right]^{T}$ is the initial condition. Note that $n_{i}$ is the dimension of the state vector $\mathbf{x}_{i}, m_{i}$ is the dimension of the control vector $\mathbf{u}_{i}$, and $p_{i}$ is the dimension of the output vector $\mathbf{y}_{i}$. 
Each component uses local control to remain stable and to meet its performance requirements. Local control means dependence only on local state or local output information, i.e., $\mathbf{u}_{i}=h_{i}\left(\mathbf{x}_{i}\right)$ or $\mathbf{u}_{i}=h_{i}\left(\mathbf{y}_{i}\right)$. In general, the local controller for an Evolving System component would have the form given by

$$
\left\{\begin{aligned}
\mathbf{u}_{i} & =h_{i}\left(\mathbf{z}_{i}\right) \\
\dot{\mathbf{z}}_{i} & =l_{i}\left(\mathbf{z}_{i}, \mathbf{y}_{i}\right)
\end{aligned}\right.
$$

where $h_{i}$ and $l_{i}$ are control operators and $\dot{\mathbf{z}}$ represents the dynamical part of the control law.

A key concept in Evolving Systems is the evolution of the connection between components, which is controlled by a connection parameter, $\epsilon$, that multiplies the forces between components. It is critical to note that connection parameters evolve independent of time. We are ignoring time in our formulation because it is assumed that joining of components is not time critical and because we are interested in studying the system as the components mate. In this framework, the evolution of the connection parameter comprises the homotopy $0 \leq \epsilon \leq 1$, not just the endpoints where $\epsilon=0$ or $\epsilon=1$. Components are unconnected when $\epsilon=0$, and connected when $\epsilon=1$. An Evolving System is fully evolved when all of the connection parameters joining the subsystem components equal 1.

Compliant forces join the components, so no degrees of freedom are lost as a consequence of two components connecting in a rigid manner. In flexible structure Evolving Systems, the compliant forces are usually springs.

The subsystem components of the Evolving System with interconnections included have the form

$$
\left\{\begin{array}{l}
\dot{\mathbf{x}}_{i}=f_{i}\left(\mathbf{x}_{i}, \mathbf{u}_{i}\right)+\sum_{j=1}^{L} \epsilon_{i j} k_{i j}(\mathbf{x}, \mathbf{u}) \\
\mathbf{y}_{i}=g_{i}\left(\mathbf{x}_{i}, \mathbf{u}_{i}\right)
\end{array}\right.
$$

where $\mathbf{x}=\left[\begin{array}{llll}\mathbf{x}_{1} & \mathbf{x}_{2} & \cdots & \mathbf{x}_{L}\end{array}\right]^{T}, \mathbf{u}=\left[\begin{array}{llll}\mathbf{u}_{1} & \mathbf{u}_{2} & \cdots & \mathbf{u}_{L}\end{array}\right]^{T}$, and the function $k_{i j}(\mathbf{x}, \mathbf{u})$ represents the interconnection between components $i$ and $j$ with connection parameter $0 \leq \epsilon_{i j} \leq 1$.

When the subsystem components join to form an Evolved System, the new entity becomes

$$
\left\{\begin{array}{l}
\dot{\mathbf{x}}=f(\mathbf{x}, \mathbf{u}) \\
\mathbf{y}=g(\mathbf{x}, \mathbf{u})
\end{array}\right.
$$

The above is the most general form of an Evolving System. Next, we introduce the framework for Evolving Systems of flexible structures.

A large and important class of nonlinear systems are linear in their input. In [2], we developed a state space description of a finite element model representation of a flexible structure Nonlinear Evolving System that is linear in its inputs. State space equations for individual components of an Evolving System, including component connections, are given by

$$
\left\{\begin{aligned}
\dot{\mathbf{x}}_{i} & =\mathbf{A}_{i}\left(\mathbf{x}_{i}\right)+\mathbf{B}_{i}\left(\mathbf{x}_{i}\right) \mathbf{u}_{i}+\sum_{j=1}^{L} \epsilon_{i j} \mathbf{A}_{i j}(\mathbf{x}) ; \mathbf{x}_{i}(0) \equiv \mathbf{x}_{i_{0}} \\
\mathbf{y}_{i} & =\mathbf{C}_{i}\left(\mathbf{x}_{i}\right)
\end{aligned}\right.
$$

where $i=1,2, \ldots, L, \mathbf{x}_{i} \equiv\left[\begin{array}{llll}x_{1}^{i} & x_{2}^{i} & \cdots & x_{n_{i}}^{i}\end{array}\right]^{T}$ is the component state vector,

$\mathbf{u}_{i} \equiv\left[\begin{array}{llll}u_{1}^{i} & u_{2}^{i} & \cdots & u_{m_{i}}^{i}\end{array}\right]^{T}$ is the control input vector, $\mathbf{y}_{i} \equiv\left[\begin{array}{llll}y_{1}^{i} & y_{2}^{i} & \cdots & y_{p_{i}}^{i}\end{array}\right]^{T}$ is the vector 
of sensed outputs, $\mathbf{x}_{i_{0}}$ is the initial condition, $\mathbf{A}_{i}\left(\mathbf{x}_{i}\right), \mathbf{B}_{i}\left(\mathbf{x}_{i}\right)$, and $\mathbf{C}_{i}\left(\mathbf{x}_{i}\right)$ are matrices that are functions of the state and have dimension $n_{i} \times n_{i}, n_{i}$ x $m_{i}$, and $p_{i} \times n_{i}$, respectively, $\mathbf{A}_{i j}(\mathbf{x})$ is the connection matrix, and $0 \leq \epsilon_{i j} \leq 1$ is the connection parameter. The connection matrix, $\mathbf{A}_{i j}(\mathbf{x})$, has dimension $n_{i}$ by $\operatorname{dim}(\mathbf{x})$, where $n_{i}$ is the dimension of the state vector $\mathbf{x}_{i}$ corresponding to component $i$ and $\operatorname{dim}(\mathbf{x}) \equiv \sum_{k=1}^{L} n_{k}$. The notation $(\mathbf{A}(\mathbf{x}), \mathbf{B}(\mathbf{x}), \mathbf{C}(\mathbf{x}))$ is used to denote an Evolving System represented by (5).

\section{Impedance-Admittance Formulation of Contact Dynamics}

In this section, we formulate the contact dynamics in Evolving Systems in terms of mechanical impedance and admittance, as first described in [9]. For many dynamical systems, the impedance-admittance form is a useful tool for modeling the contact dynamics of components [10]. The impedance of a mechanical system is determined by the equation $f=Z(v)$, where $f$ is the force exerted by the system, $v$ is the velocity of the system, and $Z$ is the impedance of the system. The admittance of a mechanical system, $Y$, is determined by the equation $v=Y(f)$ and it is the inverse of the impedance of the system, e.g., $Y \equiv Z^{-1}$. Impedance and admittance can be seen as nonlinear operators describing the relationship between the output of a mechanical system, or the force it exerts at a contact point, with the input of the system, or the velocity at the contact point.

When two components join at a point of contact, their velocities are equal and the forces exerted are equal and opposite. Thus, we can model the contact dynamics of two components mating in an Evolving System as the feedback connection of the impedance of one component with the admittance of the other component. We now introduce two nonlinear operators $Y_{1}$ and $Z_{2}$ that provide the admittance and impedance formulation of the contact dynamics of Nonlinear Evolving Systems components. These operators relate the force and velocity at the contact point of two mating components as given by the equations $\mathbf{v}_{1}=Y_{1}\left(\mathbf{f}_{1}\right)$ and $\mathbf{f}_{2}=Z_{2}\left(\mathbf{v}_{2}\right)$. In linear time-invariant systems, these operators can be easily calculated using Laplace transforms. For nonlinear components, the admittance and impedance operators cannot be easily found. However, this does not invalidate the analysis provided here, which provides a foundation for adaptive key component control and inheritance of passivity and dissipativity traits in Evolving Systems.

We can use the nonlinear impedance and admittance operators to obtain a state space representation of Evolving System components in admittance-impedance form. Following is a two component Evolving System in admittance-impedance form

$$
\left\{\begin{aligned}
\dot{\mathbf{x}}_{i} & =\mathbf{A}_{i}\left(\mathbf{x}_{i}\right)+\epsilon \mathbf{B}_{i}\left(\mathbf{x}_{i}\right) \mathbf{u}_{i}+\mathbf{B}_{i}^{A}\left(\mathbf{x}_{i}\right) \mathbf{u}_{i}^{A} \\
\mathbf{y}_{i} & =\mathbf{C}_{i}\left(\mathbf{x}_{i}\right) \\
\mathbf{y}_{i}^{A} & =\mathbf{C}_{i}^{A}\left(\mathbf{x}_{i}\right)
\end{aligned}\right.
$$

where $i=1,2, \mathbf{A}_{i}\left(\mathbf{x}_{i}\right)$ represents a component with local control, interconnections between components are represented in $\mathbf{B}_{i}\left(\mathbf{x}_{i}\right) \mathbf{u}_{i}$, control augmentation for stability of the joined system is accomplished through $\mathbf{B}_{i}^{A}\left(\mathbf{x}_{i}\right) \mathbf{u}_{i}^{A}$, and $0 \leq \epsilon \leq 1$. The Evolved System is the feedback connection of the two subsystems, i.e., where $\left(\mathbf{y}_{1}=\mathbf{u}_{2}\right)$ and $\left(\mathbf{u}_{1}=-\mathbf{y}_{2}\right)$.

\section{Adaptive Key Component Stability Restoring Controllers}

The Evolving Systems framework facilitates flexibility in the design of systems from components. Stability of an Evolving System can be lost when stable components assemble. There 
are many advantages, such as cost and reliability, to enable assembly of Evolving System components with little or no modification to the individual components. In this section, we introduce adaptive key component control to restore stability to an Evolving System by augmenting the controller on a single subsystem component, using only the input-output ports on that component. A fixed gain key component controller was first proposed in [11].

Aerospace systems are often difficult and costly to model due to their complexity and their uncertain operating environments, making them ideal candidates for adaptive control, which does not require detailed knowledge of the system parameters. Adaptive key component control was first proposed in [12]. In this approach, one component is chosen to be the key component, which is then augmented with a direct adaptive control law that only uses inputs and outputs available on that component. Since the key component controller is restoring stability to the system without using inputs or outputs on any of the other components in the Evolving System, the system needs to be controllable and observable from the key component.

Now we give equations for an Evolving System with a key component controller. Without loss of generality, we can let component 1 be the key component. We may think of component 2 as being the rest of the Evolving System to which the key component and its adaptive controller are connected. Consider a two component Nonlinear Evolving System in impedance-admittance form given by

$$
\left\{\begin{aligned}
\dot{\mathbf{x}}_{i} & =\mathbf{A}_{i}\left(\mathbf{x}_{i}\right)+\epsilon \mathbf{B}_{i}\left(\mathbf{x}_{i}\right) \mathbf{u}_{i}+\mathbf{B}_{i}^{A}\left(\mathbf{x}_{i}\right) \mathbf{u}_{i}^{A} \\
\mathbf{y}_{i} & =\mathbf{C}_{i}\left(\mathbf{x}_{i}\right) \\
\mathbf{y}_{i}^{A} & =\mathbf{C}_{i}^{A}\left(\mathbf{x}_{i}\right)
\end{aligned}\right.
$$

where $i=1,2$. Let component 1 have an adaptive key component controller with the following direct adaptive control law

$$
\left\{\begin{aligned}
\mathbf{u}_{1}^{A} & =\mathbf{G y}_{1}^{A} \\
\dot{\mathbf{G}} & =-\mathbf{y}_{1}^{A}\left(\mathbf{y}_{1}^{A}\right)^{T} \mathbf{H} ; \mathbf{H}>0
\end{aligned}\right.
$$

Note that the adaptive key component controller operates only through the input and output ports located on component 1 . The augmented control $\mathbf{u}_{\mathbf{2}}^{\mathbf{A}}\left(\mathbf{x}_{\mathbf{2}}\right)$ on component 2 would be present if additional output feedback control were needed to make the system controllable and observable from the key component or to satisfy sufficient conditions for the adaptive controller, as given in Theorem 6.1.

In the next section, we develop theory on the inheritance of dissipativity traits in Evolving Systems. This theory is used to provide sufficient conditions for adaptive key component control in Nonlinear Evolving Systems.

\section{Inheritance of Dissipativity Traits in Evolving Systems}

We say a trait, such as dissipativity, is naturally inherited by the Evolved System when all of the subsystem components have the trait and the Evolved System inherits the trait. In this section we show that two forms of dissipativity are naturally inherited in Evolving Systems. The notion of dissipativity used here is different from the dissipativity studied by other authors, e.g., $[13,14]$.

Definition 5.1. Consider a nonlinear system of the form given by

$$
\left\{\begin{array}{cc}
\dot{\mathbf{x}} & =\mathbf{A}(\mathbf{x})+\mathbf{B}(\mathbf{x}) \mathbf{u} \\
\mathbf{y} & =\mathbf{C}(\mathbf{x})
\end{array}\right.
$$


We say that this system is strictly dissipative when $\exists V(\mathbf{x})>0 \forall \mathbf{x} \neq 0$ and $V(\mathbf{0})=0$ such that $\forall \mathbf{x}$

$$
\left\{\begin{array}{l}
\nabla V \mathbf{A}(\mathbf{x}) \leq-S(\mathbf{x}) \\
\nabla V \mathbf{B}(\mathbf{x})=\mathbf{C}^{T}(\mathbf{x})
\end{array}\right.
$$

where $\nabla V \equiv$ gradient $V$ and $S(\mathbf{x})>0 \forall \mathbf{x} \neq 0$. When $S(\mathbf{x}) \equiv 0,(9)$ is a dissipative system.

The function $V(\mathbf{x})$ is referred to as an energy storage function or a Lyapunov function for (9). We can use $\nabla V$ to define the function $V(\mathbf{x})$, as follows

$$
\dot{V}(\mathbf{x}) \equiv \nabla V[\mathbf{A}(\mathbf{x})+\mathbf{B}(\mathbf{x}) \mathbf{u}]
$$

The above says that the energy storage rate is always less than the external power. This can be seen by using (10) to obtain

$$
\begin{aligned}
\dot{V}(\mathbf{x}) & \equiv \nabla V[\mathbf{A}(\mathbf{x})+\mathbf{B}(\mathbf{x}) \mathbf{u}] \\
& \leq-S(\mathbf{x})+\mathbf{C}^{T}(\mathbf{x}) \mathbf{u} \\
& =-S(\mathbf{x})+\langle\mathbf{y}, \mathbf{u}\rangle
\end{aligned}
$$

Taking $\mathbf{u} \equiv \mathbf{0}$, it is easy to see that (12) implies (10a), but not necessarily (10b). So (10) implies (12) but not conversely. If (10a) is an equality, then (10) and (12) are equivalent. If the inequalities in (10) and (12) are equalities, then the property is called strict passivity, which is described in Section 8.

Definition 5.2. Consider a nonlinear system of the form given by

$$
\left\{\begin{aligned}
\dot{\mathbf{x}} & =\mathbf{A}(\mathbf{x})+\mathbf{B}(\mathbf{x}) \mathbf{u} \\
\mathbf{y} & =\mathbf{C}(\mathbf{x})
\end{aligned}\right.
$$

We say that this system is almost strictly dissipative (ASD) when there is some output feedback, $\mathbf{u}=\mathbf{G y}+\mathbf{u}_{\mathbf{r}}$, that makes it strictly dissipative.

We now give a result on the inheritance of almost strict dissipativity in systems connected in feedback.

Theorem 5.3. Suppose we have a pair of subsystems of the form

$$
\left\{\begin{aligned}
\dot{\mathbf{x}}_{i} & =\mathbf{A}_{i}\left(\mathbf{x}_{i}\right)+\epsilon \mathbf{B}_{i}(\mathbf{x}) \mathbf{u}_{i}+\mathbf{B}_{i}^{A}(\mathbf{x}) \mathbf{u}_{i}^{A} \\
\mathbf{y}_{i} & =\mathbf{C}_{i}\left(\mathbf{x}_{i}\right) \\
\mathbf{y}_{i}^{A} & =\mathbf{C}_{i}^{A}\left(\mathbf{x}_{i}\right)
\end{aligned}\right.
$$

where $i=1,2$ and both subsystems $\left(\left[\begin{array}{l}\mathbf{u}_{1} \\ \mathbf{u}_{1}^{A}\end{array}\right],\left[\begin{array}{l}\mathbf{y}_{1} \\ \mathbf{y}_{1}^{A}\end{array}\right]\right)$ and $\left(\left[\begin{array}{l}\mathbf{u}_{2} \\ \mathbf{u}_{2}^{A}\end{array}\right],\left[\begin{array}{l}\mathbf{y}_{2} \\ \mathbf{y}_{2}^{A}\end{array}\right]\right)$ are almost strictly dissipative (strictly dissipative) with energy storage function $V_{1}\left(\mathbf{x}_{1}\right)$ and $V_{2}\left(\mathbf{x}_{2}\right)$, and

$$
\nabla V_{i} \epsilon \mathbf{B}_{i}\left(\mathbf{x}_{i}\right)=\epsilon \mathbf{C}_{i}^{T}\left(\mathbf{x}_{i}\right)
$$

Then the feedback connection of the two subsystems, where $\left(\mathbf{y}_{1}=\mathbf{u}_{2}\right)$ and $\left(\mathbf{u}_{1}=-\mathbf{y}_{2}\right)$, leaves the resulting composite system $\left(\mathbf{u}_{A} \equiv\left[\begin{array}{l}\mathbf{u}_{1}^{A} \\ \mathbf{u}_{2}^{A}\end{array}\right], \mathbf{y}_{A} \equiv\left[\begin{array}{l}\mathbf{y}_{1}^{A} \\ \mathbf{y}_{2}^{A}\end{array}\right]\right)$ almost strictly dissipative (strictly dissipative). 
Proof: We first prove the theorem for almost strict dissipativity. By the definition of almost strict dissipativity, there exists output feedback control that makes each of the subsystems strictly dissipative. It is well known [15], that output feedback can be added to a system $(\mathbf{A}(\mathbf{x}), \mathbf{B}(\mathbf{x}), \mathbf{C}(\mathbf{x}))$ to obtain

$$
\left\{\begin{array}{l}
\dot{\mathbf{x}}=\mathbf{A}(\mathbf{x})+\mathbf{B}(\mathbf{x}) \mathbf{G C}(\mathbf{x})+\mathbf{B}(\mathbf{x}) \mathbf{u}_{r} \\
\mathbf{y}=\mathbf{C}(\mathbf{x})
\end{array}\right.
$$

Let $\mathbf{u}_{i}^{A}=\mathbf{G}_{i}^{A} \mathbf{y}_{i}^{A}+\mathbf{u}_{i}^{A r}$, where $i=1,2$, be the output feedback that makes the subsystems given by (14) strictly dissipative. The subsystems with $\mathbf{u}_{i}^{A}$ defined as above are now both strictly dissipative and can be written as

$$
\left\{\begin{aligned}
\dot{\mathbf{x}}_{i} & =\mathbf{A}_{i}\left(\mathbf{x}_{i}\right)+\mathbf{B}_{i}^{A}\left(\mathbf{x}_{i}\right) \mathbf{G}_{i}^{A} \mathbf{C}_{i}^{A}\left(\mathbf{x}_{i}\right)+\epsilon \mathbf{B}_{i}(\mathbf{x}) \mathbf{u}_{i}+\mathbf{B}_{i}^{A}(\mathbf{x}) \mathbf{u}_{i}^{A r} \\
& =\mathbf{A}_{i}^{C}\left(\mathbf{x}_{i}\right)+\epsilon \mathbf{B}_{i}\left(\mathbf{x}_{i}\right) \mathbf{u}_{i}+\mathbf{B}_{i}^{A}\left(\mathbf{x}_{i}\right) \mathbf{u}_{i}^{r r} \\
\mathbf{y}_{i} & =\mathbf{C}_{i}\left(\mathbf{x}_{i}\right) \\
\mathbf{y}_{i}^{A} & =\mathbf{C}_{i}^{A}\left(\mathbf{x}_{i}\right)
\end{aligned}\right.
$$

Using the definition of strict dissipativity and the assumption (15), (17) gives us

$$
\left\{\begin{aligned}
\nabla V_{i} \mathbf{A}_{i}^{C}\left(\mathbf{x}_{i}\right) & \equiv \nabla V_{i}\left[\mathbf{A}_{i}\left(\mathbf{x}_{i}\right)+\mathbf{B}_{i}^{A}\left(\mathbf{x}_{i}\right) \mathbf{G}_{i}^{A} \mathbf{C}_{i}^{A}\left(\mathbf{x}_{i}\right)\right] \\
& \leq-S_{i}\left(\mathbf{x}_{i}\right) \\
\nabla V_{i} \in \mathbf{B}_{i}\left(\mathbf{x}_{i}\right) & =\epsilon\left(\mathbf{C}_{i}\left(\mathbf{x}_{i}\right)\right)^{T} \\
\nabla V_{i} \epsilon \mathbf{B}_{i}^{A}\left(\mathbf{x}_{i}\right) & =\epsilon\left(\mathbf{C}_{i}^{A}\left(\mathbf{x}_{i}\right)\right)^{T}
\end{aligned}\right.
$$

We can connect the two subsystems in feedback, with $\left(\mathbf{y}_{1}=\mathbf{u}_{2}\right)$ and $\left(\mathbf{u}_{1}=-\mathbf{y}_{2}\right)$. Then using the assumption (15), we obtain

$$
\begin{aligned}
\nabla V_{1} \epsilon \mathbf{B}_{1}\left(\mathbf{x}_{1}\right) \mathbf{u}_{1} & =\epsilon \mathbf{C}_{1}^{T}\left(\mathbf{x}_{1}\right)\left[-\mathbf{y}_{2}\right] \\
& =-\epsilon \mathbf{y}_{1}^{T} \mathbf{y}_{2}
\end{aligned}
$$

and

$$
\begin{aligned}
\nabla V_{2} \epsilon \mathbf{B}_{2}\left(\mathbf{x}_{2}\right) \mathbf{u}_{2} & =\epsilon \mathbf{C}_{2}^{T}\left(\mathbf{x}_{2}\right)\left[\mathbf{y}_{1}\right] \\
& =\epsilon \mathbf{y}_{2}^{T} \mathbf{y}_{1}
\end{aligned}
$$

Let $\mathbf{x} \equiv\left[\begin{array}{ll}\mathbf{x}_{1} & \mathbf{x}_{2}\end{array}\right]^{T}, \mathbf{y} \equiv\left[\begin{array}{ll}\mathbf{y}_{1}^{A} & \mathbf{y}_{2}^{A}\end{array}\right]^{T}$ and write the system from (17) in matrix form as

$$
\left\{\begin{aligned}
\dot{\mathbf{x}} & =\mathbf{A}(\mathbf{x})+\mathbf{B}(\mathbf{x}) \mathbf{u} \\
& =\left[\begin{array}{l}
\mathbf{A}_{1}^{C}\left(\mathbf{x}_{1}\right)+\epsilon \mathbf{B}_{1}\left(\mathbf{x}_{1}\right) \mathbf{u}_{1} \\
\mathbf{A}_{2}^{C}\left(\mathbf{x}_{2}\right)+\epsilon \mathbf{B}_{2}\left(\mathbf{x}_{2}\right) \mathbf{u}_{2}
\end{array}\right]+\left[\begin{array}{l}
\mathbf{B}_{1}^{A}\left(\mathbf{x}_{1}\right) \\
\mathbf{B}_{2}^{A}\left(\mathbf{x}_{2}\right)
\end{array}\right]\left[\begin{array}{l}
\mathbf{u}_{1}^{A r} \\
\mathbf{u}_{2}^{A r}
\end{array}\right] \\
\mathbf{y} & =\left[\begin{array}{l}
\mathbf{C}_{1}^{A}\left(\mathbf{x}_{1}\right) \\
\mathbf{C}_{2}^{A}\left(\mathbf{x}_{2}\right)
\end{array}\right] \\
& =\mathbf{C}(\mathbf{x})
\end{aligned}\right.
$$

Let $V=V_{1}+V_{2}$. We now can use (18)-(20) to solve

$$
\begin{aligned}
\nabla V \mathbf{A}(\mathbf{x}) & =\left[\begin{array}{ll}
\nabla V_{1} & \nabla V_{2}
\end{array}\right]\left[\begin{array}{l}
\mathbf{A}_{1}^{C}\left(\mathbf{x}_{1}\right)+\epsilon \mathbf{B}_{1}\left(\mathbf{x}_{1}\right) \mathbf{u}_{1} \\
\mathbf{A}_{2}^{C}\left(\mathbf{x}_{2}\right)+\epsilon \mathbf{B}_{2}\left(\mathbf{x}_{2}\right) \mathbf{u}_{2}
\end{array}\right] \\
& =\nabla V_{1} \mathbf{A}_{1}^{C}\left(\mathbf{x}_{1}\right)+\nabla V_{1} \epsilon \mathbf{B}_{1}\left(x_{1}\right) \mathbf{u}_{1}+\nabla V_{2} \mathbf{A}_{2}^{C}\left(\mathbf{x}_{2}\right)+\nabla V_{2} \epsilon \mathbf{B}_{2}\left(x_{2}\right) \mathbf{u}_{2} \\
& \leq-S_{1}\left(\mathbf{x}_{1}\right)+\left(-\epsilon \mathbf{y}_{1}^{T} \mathbf{y}_{2}\right)-S_{2}\left(\mathbf{x}_{2}\right)+\epsilon \mathbf{y}_{2}^{T} \mathbf{y}_{1} \\
& =-\left(S_{1}\left(\mathbf{x}_{1}\right)+S_{2}\left(\mathbf{x}_{2}\right)\right) \\
& =-S(\mathbf{x})
\end{aligned}
$$


and

$$
\begin{aligned}
\nabla V \mathbf{B}(\mathbf{x}) & =\left[\begin{array}{ll}
\nabla V_{1} & \nabla V_{2}
\end{array}\right]\left[\begin{array}{cc}
\mathbf{B}_{1}^{A}\left(\mathbf{x}_{1}\right) & 0 \\
0 & \mathbf{B}_{1}^{A}\left(\mathbf{x}_{2}\right)
\end{array}\right] \\
& =\left[\begin{array}{l}
\mathbf{C}_{1}^{A}\left(\mathbf{x}_{1}\right) \\
\mathbf{C}_{2}^{A}\left(\mathbf{x}_{2}\right)
\end{array}\right]^{T} \\
& =\mathbf{C}^{T}(\mathbf{x})
\end{aligned}
$$

Therefore the composite system given by $\left(\mathbf{u}_{A} \equiv\left[\begin{array}{l}\mathbf{u}_{1}^{A} \\ \mathbf{u}_{2}^{A}\end{array}\right], \mathbf{y}_{A} \equiv\left[\begin{array}{l}\mathbf{y}_{1}^{A} \\ \mathbf{y}_{2}^{A}\end{array}\right]\right)$ is made strictly dissipative with output feedback $\left[\begin{array}{l}\mathbf{u}_{1}^{A} \\ \mathbf{u}_{2}^{A}\end{array}\right] \equiv\left[\begin{array}{cc}\mathbf{G}_{1}^{A} & 0 \\ 0 & \mathbf{G}_{2}^{A}\end{array}\right]\left[\begin{array}{l}\mathbf{y}_{1}^{A} \\ \mathbf{y}_{2}^{A}\end{array}\right]+\left[\begin{array}{l}\mathbf{u}_{1}^{A r} \\ \mathbf{u}_{2}^{A r}\end{array}\right]$. Hence, the composite system is almost strictly dissipative.

We now prove the theorem for strict dissipativity. Since the two subsystems are strictly dissipative, we can let $\mathbf{G}_{i}^{A} \equiv 0$ in the preceding proof. Then the proof for this result follows directly and the composite system is strictly dissipative.

A consequence of Theorem 5.3 is that two component Nonlinear Evolving Systems with components that are either both almost strictly dissipative or strictly dissipative from an admittanceimpedance point of view, inherit these properties from their subsystem components. Thus almost strict dissipativity and strict dissipativity are naturally inherited traits in Nonlinear Evolving Systems.

\section{Sufficient Conditions for Adaptive Key Component Control}

We can now give sufficient conditions for the use of an adaptive key component controller to restore stability in Nonlinear Evolving Systems.

Theorem 6.1. Consider a two component nonlinear time-invariant Evolving System given by

$$
\left\{\begin{aligned}
\dot{\mathbf{x}}_{i} & =\mathbf{A}_{i}\left(\mathbf{x}_{i}\right)+\epsilon \mathbf{B}_{i}\left(\mathbf{x}_{i}\right) \mathbf{u}_{i}+\mathbf{B}_{i}^{A}\left(\mathbf{x}_{i}\right) \mathbf{u}_{i}^{A} \\
\mathbf{y}_{i} & =\mathbf{C}_{i}\left(\mathbf{x}_{i}\right)
\end{aligned}\right.
$$

where $i=1,2$ with energy storage functions $V_{1}\left(\mathbf{x}_{1}\right)$ and $V_{2}\left(\mathbf{x}_{2}\right)$. Let component 1 have an adaptive key component controller with the following direct adaptive control law

$$
\left\{\begin{aligned}
\mathbf{u}_{1}^{A} & =\mathbf{G y}_{1}^{A} \\
\dot{\mathbf{G}} & =-\mathbf{y}_{1}^{A}\left(\mathbf{y}_{1}^{A}\right)^{T} \mathbf{H} ; \mathbf{H}>0
\end{aligned}\right.
$$

Assume that $V_{1}$ and $V_{2}$ are positive $\forall \mathbf{x} \neq 0$, equal to 0 when $\mathbf{x}=\mathbf{0}$, and radially unbounded, that $(\mathbf{A}(\mathbf{x}), \mathbf{B}(\mathbf{x}), \mathbf{C}(\mathbf{x}))$ are continuous functions of $\mathbf{x}$, and that $S(\mathbf{x})$ is positive $\forall \mathbf{x} \neq 0$ and has continuous partial derivatives in $\mathbf{x}$. Furthermore, assume:

1. Component 2, given by $\left(\mathbf{u}_{2}, \mathbf{y}_{2}\right)$, is strictly dissipative and in impedance form;

2. Component 1, given by $\left(\mathbf{u}_{1}^{A}, \mathbf{y}_{1}^{A}\right)$, is almost strictly dissipative;

3. Component 1 , given by $\left(\mathbf{u}_{1}, \mathbf{y}_{1}\right)$, is in admittance form.

Then the adaptive key component controller given by (25) that joins component 1 with component 2 produces global asymptotic state stability, i.e., $\mathbf{x} \equiv\left[\begin{array}{ll}\mathbf{x}_{1} & \mathbf{x}_{2}\end{array}\right]^{T} \rightarrow \mathbf{0}$ as $t \rightarrow \infty$ with bounded adaptive gains and the outputs $\mathbf{y}_{i}=\mathbf{C}_{i}\left(\mathbf{x}_{i}\right) \rightarrow \mathbf{0}$ as $t \rightarrow \infty$. 
Proof: Since the physical connection of component 1 to component 2 is equivalent to the feedback connection $\mathbf{u}_{1}=-\mathbf{y}_{2}$ and $\mathbf{u}_{2}=\mathbf{y}_{1}$, by Theorem 5.3 we have that the closed-loop system $\left(\mathbf{u}_{1}^{A}, \mathbf{y}_{1}^{A}\right)$ below is almost strictly dissipative.

$$
\left\{\begin{aligned}
\dot{\mathbf{x}}_{1} & =\mathbf{A}_{1}\left(\mathbf{x}_{1}\right)-\epsilon \mathbf{B}_{1}\left(\mathbf{x}_{1}\right) \mathbf{C}_{2}\left(\mathbf{x}_{2}\right)+\mathbf{B}_{1}^{A}\left(\mathbf{x}_{1}\right) \mathbf{u}_{1}^{A} \\
\dot{\mathbf{x}}_{2} & =\mathbf{A}_{2}\left(\mathbf{x}_{2}\right)+\epsilon \mathbf{B}_{2}\left(\mathbf{x}_{2}\right) \mathbf{C}_{1}\left(\mathbf{x}_{1}\right) \\
\mathbf{y}_{1}^{A} & =\mathbf{C}_{1}^{A}\left(\mathbf{x}_{1}\right)
\end{aligned}\right.
$$

Rewrite (25), using $\mathbf{G}_{1}^{*}$ constant to get

$$
\left\{\begin{aligned}
\mathbf{u}_{1}^{A} & =\mathbf{G}_{1} \mathbf{y}_{1}^{A}=\mathbf{G}_{1}^{*} \mathbf{y}_{1}^{A}+\Delta \mathbf{G}_{1} \mathbf{y}_{1}^{A} \\
\Delta \dot{\mathbf{G}}_{1} & =\dot{\mathbf{G}}_{1}=-\mathbf{y}_{1}^{A}\left(\mathbf{y}_{1}^{A}\right)^{T} \mathbf{h}_{1}
\end{aligned}\right.
$$

where $\Delta \mathbf{G}_{1} \equiv \mathbf{G}_{1}-\mathbf{G}_{1}^{*}$ and $\mathbf{h}_{1}>0$. Combine (26) and (27) to obtain

$$
\left\{\begin{aligned}
\dot{\mathbf{x}}_{1} & =\mathbf{A}_{1}^{C}\left(\mathbf{x}_{1}\right)-\epsilon \mathbf{B}_{1}\left(\mathbf{x}_{1}\right) \mathbf{C}_{2}\left(\mathbf{x}_{2}\right)+\mathbf{B}_{1}^{A}\left(\mathbf{x}_{1}\right) \omega_{1}^{A} \\
\dot{\mathbf{x}}_{2} & =\mathbf{A}_{2}\left(\mathbf{x}_{2}\right)+\epsilon \mathbf{B}_{2}\left(\mathbf{x}_{2}\right) \mathbf{C}_{1}\left(\mathbf{x}_{1}\right) \\
\mathbf{y}_{1}^{A} & =\mathbf{C}_{1}^{A}\left(\mathbf{x}_{1}\right)
\end{aligned}\right.
$$

where $\mathbf{A}_{1}^{C}\left(\mathbf{x}_{1}\right) \equiv \mathbf{A}_{1}\left(\mathbf{x}_{1}\right)+\mathbf{B}_{1}^{A}\left(\mathbf{x}_{1}\right) \mathbf{G}_{1}^{*} \mathbf{C}_{1}^{A}\left(\mathbf{x}_{1}\right), \omega_{1}^{A} \equiv \Delta \mathbf{G}_{1} \mathbf{y}_{1}^{A}$, and $0 \leq \epsilon \leq 1$.

Let $V=V_{1}+V_{2}$. We now have

$$
\dot{V}=-S(\mathbf{x})+\left\langle\mathbf{y}_{1}^{A}, \omega_{1}^{A}\right\rangle
$$

Form $V_{G} \equiv \frac{1}{2} \operatorname{tr}\left(\Delta \mathbf{G}_{1} \mathbf{h}_{1}^{-1} \Delta \mathbf{G}_{1}^{T}\right)$ to obtain

$$
\begin{aligned}
\dot{V}_{G} & \equiv \operatorname{tr}\left(\Delta \dot{\mathbf{G}}_{1} \mathbf{h}_{1}^{-1} \Delta \mathbf{G}_{1}^{T}\right) \\
& =-\operatorname{tr}\left(\mathbf{y}_{1}^{A}\left(\mathbf{y}_{1}^{A}\right)^{T} \mathbf{h}_{1} \mathbf{h}_{1}^{-1} \Delta \mathbf{G}_{1}^{T}\right) \\
& =-\operatorname{tr}\left(\mathbf{y}_{1}^{A}\left(\omega_{1}^{A}\right)^{T}\right) \\
& =-\left\langle\mathbf{y}_{1}^{A}, \omega_{1}^{A}\right\rangle
\end{aligned}
$$

Define $V(\mathbf{x}, \Delta \mathbf{G}) \equiv V(\mathbf{x})+V_{G}(\Delta \mathbf{G})$. Then from (29) and (30), we have

$$
\begin{aligned}
\dot{V}(\mathbf{x}, \Delta \mathbf{G}) & \equiv \dot{V}(\mathbf{x})+\dot{V}_{G}(\Delta \mathbf{G}) \\
& =-S(\mathbf{x})+\left\langle\mathbf{y}_{1}^{A}, \omega_{1}^{A}\right\rangle-\left\langle\mathbf{y}_{1}^{A}, \omega_{1}^{A}\right\rangle \\
& =-S(\mathbf{x}) \leq 0
\end{aligned}
$$

This guarantees that all trajectories $(\mathbf{x}, \Delta \mathbf{G})$ are bounded. If $\dot{V}(\mathbf{x}, \Delta \mathbf{G})$ is uniformly continuous or $\ddot{V}(\mathbf{x}, \Delta \mathbf{G})$ is bounded, then Barbalat's Lemma [16] yields: $S(\mathbf{x}) \rightarrow 0$, as $t \rightarrow \infty$, and the positivity and continuity of $S(\mathbf{x})$ imply that $\mathbf{x} \equiv\left[\begin{array}{ll}\mathbf{x}_{1} & \mathbf{x}_{2}\end{array}\right]^{T} \rightarrow 0$ as $t \rightarrow \infty$.

Consider

$$
\begin{aligned}
& \ddot{V}(\mathbf{x}, \Delta \mathbf{G})=-\dot{S}(\mathbf{x}) \\
& \leq|\dot{S}(\mathbf{x})| \\
& =\left|\frac{\partial S(\mathbf{x})}{\partial \mathbf{x}} \dot{\mathbf{x}}\right| \\
& \leq \quad\left\|\frac{\partial S(\mathbf{x})}{\partial \mathbf{x}}\right\|_{\|\dot{\mathbf{x}}\|} \\
& \leq \quad \frac{\partial S(\mathbf{x})}{\partial \mathbf{x}} \|\left[\|\mathbf{A}(\mathbf{x})\|+\|\mathbf{B}(\mathbf{x})\|\left\|\omega_{1}^{A}\right\|\right] \\
& \leq \frac{\partial S(\mathbf{x})}{\partial \mathbf{x}} \| \underset{9}{\left[\|\mathbf{A}(\mathbf{x})\|+\|\mathbf{B}(\mathbf{x})\|\left\|\Delta \mathbf{G}_{1}\left|\left\|\mid \mathbf{C}_{1}^{A}\left(\mathbf{x}_{1}\right)\right\|\right]\right.\right.}
\end{aligned}
$$


which is bounded because $(\mathbf{x}, \Delta \mathbf{G})$ is bounded, $S(\mathbf{x})$ has continuous partial derivatives, and $(\mathbf{A}(\mathbf{x}), \mathbf{B}(\mathbf{x}), \mathbf{C}(\mathbf{x}))$ are continuous, and a continuous function of a bounded $\mathbf{x}(t)$ in $\mathbb{R}^{N}$ is also bounded in $t$. Therefore, $\mathbf{y}_{i}=\mathbf{C}_{i}\left(\mathbf{x}_{i}\right) \rightarrow 0$ as $t \rightarrow 0$.

\section{Special Case: Internal State Nonlinearity}

Here we look at a special case of the above theory when the only nonlinearity is in the internal state structure of each component. This means that the Evolving System from (7) can be written as

$$
\left\{\begin{aligned}
\dot{\mathbf{x}}_{i} & =\mathbf{A}_{i} \mathbf{x}_{i}+\mathbf{h}_{i}\left(\mathbf{x}_{i}\right)+\epsilon \mathbf{B}_{i} \mathbf{u}_{i}+\mathbf{B}_{i}^{A} \mathbf{u}_{i}^{A} \\
\mathbf{y}_{i} & =\mathbf{C}_{i} \mathbf{x}_{i} \\
\mathbf{y}_{i}^{A} & =\mathbf{C}_{i}^{A} \mathbf{x}_{i}
\end{aligned}\right.
$$

where $i=1,2$. The nonlinearities are assumed to satisfy $\mathbf{h}_{i}(\mathbf{0})=\mathbf{0}$ and a Lipschitz continuity condition given by

$$
\left\|\mathbf{h}_{i}(\mathbf{x})-\mathbf{h}_{i}(\mathbf{y})\right\| \leq \mu_{i}\|\mathbf{x}-\mathbf{y}\|
$$

$\forall \mathbf{x}, \mathbf{y}$ with $\mu_{i}>0$.

We can choose quadratic Lyapunov functions of the form $V(\mathbf{x}) \equiv \frac{1}{2} \mathbf{x}_{i}^{T} \mathbf{P}_{i} \mathbf{x}_{i}$. We assume the linear part of each component $\left(\mathbf{A}_{i}, \mathbf{B}_{i}, \mathbf{C}_{i}\right)$ is almost strict positive real (ASPR), i.e., $\exists \mathbf{G}_{i}^{*}$ such that $\mathbf{A}_{i}^{C} \equiv \mathbf{A}_{i}+\mathbf{B}_{i} \mathbf{G}_{i}^{*} \mathbf{C}_{i}$ satisfies

$$
\left\{\begin{array}{l}
\left(\mathbf{A}_{i}^{C}\right)^{T} \mathbf{P}_{i}+\mathbf{P}_{i} \mathbf{A}_{i}^{C}=-\mathbf{Q}_{i} \\
\mathbf{P}_{i} \mathbf{B}_{i}^{A}=\left(\mathbf{C}_{i}^{A}\right)^{T}
\end{array}\right.
$$

with $\mathbf{P}_{i}, \mathbf{Q}_{i}$ both positive definite. In addition, we assume that

$$
\mathbf{P}_{i} \mathbf{B}_{i}=\mathbf{C}_{i}^{T}
$$

with the same $\mathbf{P}_{i}>0$. Then, from (35) - (36) we have

$$
\left\{\begin{aligned}
\nabla V_{i} \mathbf{A}_{i}^{C}\left(\mathbf{x}_{i}\right) & =\mathbf{x}_{i}^{T} \mathbf{P}_{i}\left[\left(\mathbf{A}_{i}+\mathbf{B}_{i}^{A} \mathbf{G}_{i}^{*} \mathbf{C}_{i}^{A}\right) \mathbf{x}_{i}+\mathbf{h}_{i}\left(\mathbf{x}_{i}\right)+\epsilon \mathbf{B}_{i} \mathbf{u}_{i}\right] \\
& =-\frac{1}{2} \mathbf{x}_{i}^{T} \mathbf{Q}_{i} \mathbf{x}_{i}+\mathbf{x}_{i}^{T} \mathbf{P}_{i} \mathbf{h}_{i}\left(\mathbf{x}_{i}\right)+\epsilon \mathbf{y}_{i}^{T} \mathbf{u}_{i} \\
\nabla V_{i} \mathbf{B}_{i}^{A}\left(\mathbf{x}_{i}\right) & =\mathbf{x}_{i}^{T} \mathbf{P}_{i} \mathbf{B}_{i}^{A}=\left(\mathbf{C}_{i}^{A} x_{i}\right)^{T}
\end{aligned}\right.
$$

Now, from (34) and the Cauchy-Schwarz inequality, we have

$$
\begin{aligned}
-\frac{1}{2} \mathbf{x}_{i}^{T} \mathbf{Q}_{i} \mathbf{x}_{i}+\mathbf{x}_{i}^{T} \mathbf{P}_{i} \mathbf{h}_{i}\left(\mathbf{x}_{i}\right) & \leq-\left(\frac{\lambda_{\min }\left(\mathbf{Q}_{i}\right)}{2}-\lambda_{\max }\left(\mathbf{P}_{i}\right) \mu\right) \mathbf{x}_{i}^{T} \mathbf{x}_{i} \\
& =-\gamma_{i}\left\|\mathbf{x}_{i}\right\|^{2} \\
& \equiv-S_{i}\left(\mathbf{x}_{i}\right)
\end{aligned}
$$

Therefore, (37) becomes

$$
\left\{\begin{aligned}
\nabla V_{i} \mathbf{A}_{i}^{C}\left(\mathbf{x}_{i}\right) & =\mathbf{x}_{i}^{T} \mathbf{P}_{i}\left[\left(\mathbf{A}_{i}+\mathbf{B}_{i}^{A} \mathbf{G}_{i}^{*} \mathbf{C}_{i}^{A}\right) \mathbf{x}_{i}+\mathbf{h}_{i}\left(\mathbf{x}_{i}\right)+\epsilon \mathbf{B}_{i} \mathbf{u}_{i}\right] \\
& \leq-S_{i}\left(\mathbf{x}_{i}\right)+\epsilon\left\langle\mathbf{y}_{i}, \mathbf{u}_{i}\right\rangle \\
\nabla V_{i} \mathbf{B}_{i}^{A}\left(\mathbf{x}_{i}\right) & =\mathbf{x}_{i}^{T} \mathbf{P}_{i} \mathbf{B}_{i}^{A}=\left(\mathbf{C}_{i}^{A} x_{i}\right)^{T}
\end{aligned}\right.
$$

with $S_{i}\left(\mathbf{x}_{i}\right) \equiv \gamma_{i}\left\|\mathbf{x}_{i}\right\|^{2}>0 \forall \mathbf{x}_{i} \neq 0$ when $\gamma_{i} \equiv \frac{\lambda_{\min }\left(\mathbf{Q}_{i}\right)}{2}-\lambda_{\max }\left(\mathbf{P}_{i}\right) \mu_{i}>0$. From this analysis, we have the following result: 
Theorem 7.1. If the linear part, $\left(\mathbf{A}_{i}, \mathbf{B}_{i}^{A}, \mathbf{C}_{i}^{A}\right)$, of (33) is ASPR, then (36) holds, and the Lipschitz constant in (34) satisfies the following

$$
0<\mu_{i}<\frac{\lambda_{\min }\left(\mathbf{Q}_{i}\right)}{2 \lambda_{\max }\left(\mathbf{P}_{i}\right)}
$$

Then, when component 1 is joined with component 2, the adaptive key component controller given by (8) maintains closed-loop stability.

Proof: From (40), we obtain (39) and we can apply Theorem 6.1 to achieve the result.

\section{Inheritance of Passivity Traits in Evolving Systems}

In this section we give results describing the inheritance of strict and almost strict passivity in Nonlinear Evolving Systems.

Definition 8.1. Consider a nonlinear system of the form given by

$$
\left\{\begin{aligned}
\dot{\mathbf{x}} & =\mathbf{A}(\mathbf{x})+\mathbf{B}(\mathbf{x}) \mathbf{u} \\
\mathbf{y} & =\mathbf{C}(\mathbf{x})
\end{aligned}\right.
$$

We say that this system is strictly passive when $\exists V(\mathbf{x})>0 \forall \mathbf{x} \neq 0$ and $V(\mathbf{0})=0$ such that

$$
\dot{V}(\mathbf{x})=\langle\mathbf{u}, \mathbf{y}\rangle-S(\mathbf{x})
$$

with $S(\mathbf{x})>0 \forall \mathbf{x} \neq 0$.

Definition 8.2. Consider a nonlinear system of the form given by

$$
\left\{\begin{array}{l}
\dot{\mathbf{x}}=\mathbf{A}(\mathbf{x})+\mathbf{B}(\mathbf{x}) \mathbf{u} \\
\mathbf{y}=\mathbf{C}(\mathbf{x})
\end{array}\right.
$$

We say that this system is almost strictly passive (ASP) when there is some output feedback, $\mathbf{u}=\mathbf{G y}+\mathbf{u}_{\mathbf{r}}$, that makes it strictly passive.

We state the following result without proof. The result is an obvious consequence of Theorem 5.3 and a well known result about passivity and systems connected in feedback [16].

Theorem 8.3. Suppose we have a pair of subsystems of the form

$$
\left\{\begin{aligned}
\dot{\mathbf{x}}_{i} & =\mathbf{A}_{i}\left(\mathbf{x}_{i}\right)+\mathbf{B}_{i}\left(\mathbf{x}_{i}\right) \mathbf{u}_{i}+\mathbf{B}_{i}^{A}\left(\mathbf{x}_{i}\right) \mathbf{u}_{i}^{A} \\
\mathbf{y}_{i} & =\mathbf{C}_{i}\left(\mathbf{x}_{i}\right) \\
\mathbf{y}_{i}^{A} & =\mathbf{C}_{i}^{A}\left(\mathbf{x}_{i}\right)
\end{aligned}\right.
$$

where $i=1,2$ and both subsystems $\left(\left[\begin{array}{l}\mathbf{u}_{1} \\ \mathbf{u}_{1}^{A}\end{array}\right],\left[\begin{array}{l}\mathbf{y}_{1} \\ \mathbf{y}_{1}^{A}\end{array}\right]\right)$ and $\left(\left[\begin{array}{l}\mathbf{u}_{2} \\ \mathbf{u}_{2}^{A}\end{array}\right],\left[\begin{array}{l}\mathbf{y}_{2} \\ \mathbf{y}_{2}^{A}\end{array}\right]\right)$ are almost strictly passive (strictly passive) with energy storage functions $V_{1}\left(\mathbf{x}_{1}\right)$ and $V_{2}\left(\mathbf{x}_{2}\right)$. Then the feedback connection of the two subsystems, where $\left(\mathbf{y}_{1}=\mathbf{u}_{2}\right)$ and $\left(\mathbf{u}_{1}=-\mathbf{y}_{2}\right)$, leaves the resulting composite system $\left(\mathbf{u}_{A} \equiv\left[\begin{array}{l}\mathbf{u}_{1}^{A} \\ \mathbf{u}_{2}^{A}\end{array}\right], \mathbf{y}_{A} \equiv\left[\begin{array}{l}\mathbf{y}_{1}^{A} \\ \mathbf{y}_{2}^{A}\end{array}\right]\right)$ almost strictly passive (strictly passive). 
Theorem 8.3 can be used to show that two component Nonlinear Evolving Systems with components that are either both almost strictly passive or strictly passive from an admittanceimpedance point of view, inherit these properties from their subsystem components. Thus strict passivity and almost strict passivity are naturally inherited traits in Nonlinear Evolving Systems.

All of the preceding results assume that the Lyapunov function, $V(\mathbf{x})$, is defined on the entire domain, $\mathbb{R}^{n}$, of the system. Thus all the stability and dissipativity results and Theorem 6.1 are global results. However, the Lyapunov function, $V(\mathbf{x})$, might only be defined on a neighborhood $N_{i}\left(0, r_{i}\right) \equiv\left\{\frac{\mathbf{x}_{i}}{\left\|\mathbf{x}_{i}\right\|}<r_{i}\right\}$ of the origin, in which case the results could only be local at the best.

Using Lemma 1 from [17], $\exists \delta>0$ such that if the initial conditions of the system are close enough to the origin, i.e., within $N_{\delta}=(0, \delta)$, then the trajectories are guaranteed to stay in the neighborhood of the origin for which the Lyapunov function is defined. In such a case, then the results would be local. For instance, if the Lyapunov function $V(\mathbf{x})$ in Theorem 6.1 only has the assumed properties on a neighborhood $N_{i}\left(0, r_{i}\right) \equiv\left\{\frac{\mathbf{x}_{i}}{\left\|\mathbf{x}_{i}\right\|}<r_{i}\right\}$ of the origin and the trajectories all remain inside the neighborhood, then the stability is locally asymptotic to the origin. In that case, Theorem 6.1 gives the result that a Nonlinear Evolving System with an adaptive key component controller as given by (25) will have bounded gains and locally asymptotic state tracking.

\section{Equivalence of Traits in LTI Evolving Systems}

In this section we give equivalence results for some properties of LTI Evolving Systems.

Theorem 9.1. If a nonlinear system given by $(\mathbf{A}(\mathbf{x}), \mathbf{B}(\mathbf{x}), \mathbf{C}(\mathbf{x}))$ is strictly passive, then it is strictly dissipative.

Proof: Let $V(\mathbf{x})$ be the energy storage function for the system $(\mathbf{A}(\mathbf{x}), \mathbf{B}(\mathbf{x}), \mathbf{C}(\mathbf{x}))$. By the definition of the gradient and strict passivity, we have

$$
\begin{aligned}
\dot{V}(x) & \equiv \nabla V \mathbf{A}(\mathbf{x})+\nabla \mathbf{B}(\mathbf{x}) \mathbf{u} \\
& =-S(\mathbf{x})+\mathbf{y}^{T} \mathbf{u}
\end{aligned}
$$

Let $\mathbf{u} \equiv \mathbf{0}$. Then (45) becomes

$$
\dot{V}(\mathbf{x})=\nabla V \mathbf{A}(\mathbf{x})=-S(\mathbf{x}) \leq-S(x)
$$

Thus, $\mathbf{y}^{T} \mathbf{u}=\nabla V \mathbf{B}(\mathbf{x}) \mathbf{u} \forall \mathbf{u}$. Since $\mathbf{y}=\mathbf{C}(\mathbf{x})$, this implies that $\nabla V \mathbf{B}(\mathbf{x})=\mathbf{C}^{T}(\mathbf{x})$. Therefore, the system $(\mathbf{A}(\mathbf{x}), \mathbf{B}(\mathbf{x}), \mathbf{C}(\mathbf{x}))$ is strictly dissipative.

Theorem 9.2. A LTI system given by $(\mathbf{A}, \mathbf{B}, \mathbf{C})$ is strictly dissipative iff it is strictly passive.

Proof: First we show that a LTI system that is strictly dissipative is strictly passive. Since $(\mathbf{A}, \mathbf{B}, \mathbf{C})$ is LTI and strictly dissipative, by the Kalman-Yacubovic Lemma [16], $\exists$ quadratic Lyapunov function $V(\mathbf{x})$ such that $\forall \mathbf{x} \in \mathbb{R}^{n}$

$$
\begin{aligned}
\nabla V \mathbf{A x} & =\frac{1}{2} \mathbf{x}^{T}\left(\mathbf{P A}+\mathbf{A}^{T} \mathbf{P}\right) \mathbf{x} \leq-S(\mathbf{x}) \\
\nabla V \mathbf{B} & =\mathbf{x}^{T} \mathbf{P B}=\mathbf{x}^{T} \mathbf{C}=\mathbf{y}^{T}
\end{aligned}
$$


Choose $S(\mathbf{x}) \equiv \frac{1}{2} \mathbf{x}^{T} \mathbf{Q x}$ with $\mathbf{Q}>0$. Then we have

$$
\begin{aligned}
\nabla V \mathbf{A x} & =\frac{1}{2} \mathbf{x}^{T}\left(\mathbf{P A}+\mathbf{A}^{T} \mathbf{P}\right) \mathbf{x} \leq-\frac{1}{2} \mathbf{x}^{T} \mathbf{Q} \mathbf{x} \\
& =\mathbf{P A}+\mathbf{A}^{T} \mathbf{P} \leq-\mathbf{Q}
\end{aligned}
$$

Let $\mathbf{W} \equiv \mathbf{P A}+\mathbf{A}^{T} \mathbf{P}$, then $\mathbf{W}$ is symmetric and $\mathbf{W} \leq-\mathbf{Q}$. Then $\exists \mathbf{H}, \mathbf{H}$ symmetric such that $\mathbf{W}+\mathbf{H}=-\mathbf{Q}$. This can be seen if we let $\mathbf{H} \equiv-(\mathbf{Q}+\mathbf{W}) \geq 0$. Then $\mathbf{Q}+\mathbf{H}>0$, because $\mathbf{Q}>0$. So

$$
\nabla V \mathbf{A x}=\mathbf{P A}+\mathbf{A}^{T} \mathbf{P}=-(\mathbf{Q}+\mathbf{H})
$$

Now we can write

$$
\dot{V}(\mathbf{x})=\nabla V \mathbf{A x}+\nabla V \mathbf{B u}=-(\mathbf{Q}+\mathbf{H})+\mathbf{y}^{T} \mathbf{u}
$$

$\forall \mathbf{x} \in \mathbb{R}^{n}$. Hence, $(\mathbf{A}, \mathbf{B}, \mathbf{C})$ is strictly passive. The converse is true by Theorem 9.1.

Theorem 9.3. A LTI system given by $(\mathbf{A}, \mathbf{B}, \mathbf{C})$ is strict positive real (SPR) iff it is strictly passive.

Proof: First we show that if $(\mathbf{A}, \mathbf{B}, \mathbf{C})$ is SPR, then it is strictly passive. Since $(\mathbf{A}, \mathbf{B}, \mathbf{C})$ is $\mathrm{SPR}$, the Kalman-Yacubovic Lemma [16] implies that $\exists \epsilon>0$ such that

$$
\left\{\begin{array}{l}
(\mathbf{A}+\epsilon \mathbf{I})^{T} \mathbf{P}+\mathbf{P}(\mathbf{A}+\epsilon \mathbf{I})=-\mathbf{Q} \\
\mathbf{P B}=\mathbf{C}^{T}
\end{array}\right.
$$

with $\mathbf{Q} \geq 0$ and $\mathbf{P}>0$. We can rearrange (51) to obtain

$$
\left\{\begin{array}{l}
\mathbf{A}^{T} \mathbf{P}+\mathbf{P A}=-(\mathbf{Q}+2 \epsilon \mathbf{P}) \\
\mathbf{P B}=\mathbf{C}^{T}
\end{array}\right.
$$

Since $\mathbf{P}>0$ and $\mathbf{Q} \geq 0$, then $\mathbf{W}(\epsilon) \equiv \mathbf{Q}+2 \epsilon \mathbf{P}>0$. Choose $V(\mathbf{x}) \equiv \frac{1}{2} \mathbf{x}^{T} \mathbf{P x}$ with $\mathbf{P}$ chosen as in (51) and $\mathbf{P}>0$. The time derivative along any state trajectory of $V(\mathbf{x})$ is given by

$$
\begin{aligned}
\dot{V}(\mathbf{x}) & =\frac{1}{2}\left(\mathbf{x}^{T} \mathbf{P A} \dot{\mathbf{x}}+\dot{\mathbf{x}}^{T} \mathbf{P B u x}\right) \\
& =\frac{1}{2} \mathbf{x}^{T}\left(\mathbf{A}^{T} \mathbf{P}+\mathbf{P A}\right) \mathbf{x}+\mathbf{x}^{T} \mathbf{P B u} \\
& =-\frac{1}{2} \mathbf{x}^{T} \mathbf{W}(\epsilon) \mathbf{x}+(\mathbf{C x})^{T} \mathbf{u} \\
& =-S(\mathbf{x})+\mathbf{y}^{T} \mathbf{u}
\end{aligned}
$$

Therefore $(\mathbf{A}, \mathbf{B}, \mathbf{C})$ is strictly passive.

We now show that if $(\mathbf{A}, \mathbf{B}, \mathbf{C})$ is strictly passive, then it is SPR. Since $(\mathbf{A}, \mathbf{B}, \mathbf{C})$ is strictly passive, we have that $V(\mathbf{x})=-S(\mathbf{x})+\mathbf{y}^{T} \mathbf{u}$ with $S(\mathbf{x})>0$. Choose $S(\mathbf{x}) \equiv \mathbf{W}(\epsilon)$, with $\mathbf{P}$ and $\mathbf{Q}$ as defined in (51). Then all of the previous arguments can be reversed, giving the desired result.

We have shown that for LTI systems, strict positive real is equivalent to strict passivity.

A system that is almost strict positive real (ASPR) is defined to be one that can be made strict positive real with output feedback. Hence, for LTI systems, the above results give us that almost strict positive real is equivalent to almost strict passivity. Likewise, the above results give us that almost strict passivity is equivalent to almost strict dissipativity in LTI systems.

The equivalence results of the above properties in LTI systems also apply to LTI Evolving Systems. Hence, for LTI Evolving Systems, we can substitute any of the equivalent properties into Theorem 6.1. 


\section{Illustrative Example of Nonlinear Evolving System with an Adaptive Key Component Controller}

We now give an illustrative example of an adaptive key component controller restoring stability in a Nonlinear Evolving System where each of the components is actively controlled and stable, but the Evolving System fails to inherit the stability of the components. Consider a fully actuated, fully sensed three mass Evolving System. Component 1 has only one mass. The dynamical equations for component 1 are

$$
\left\{\begin{aligned}
m_{1} \ddot{q}_{1} & =u_{1} \\
\mathbf{y}_{\mathbf{1}} & =\left[\begin{array}{ll}
q_{1} & \dot{q}_{1}
\end{array}\right]^{T}
\end{aligned}\right.
$$

where $m_{1}=30$ is the mass of mass $1, q_{1}$ is the displacement of mass 1 , and $u_{1}=-(0.9 s+0.1) q_{1}$ is the local controller for component 1 , where $s$ is the Laplace variable.

Component 2 has two masses connected by a nonlinear spring. The dynamical equations for component 2 are

$$
\left\{\begin{aligned}
m_{2} \ddot{q}_{2} & =u_{2}-k_{23}\left(q_{2}-q_{3}\right) \\
m_{3} \ddot{q}_{3} & =u_{2}-\left[k_{23}\left(q_{3}-q_{2}\right)+\mu_{23} \sin \left(q_{3}-q_{2}\right)\right] \\
\mathbf{y}_{\mathbf{2}} & =\left[\begin{array}{ll}
q_{2} & \dot{q}_{2}
\end{array}\right]^{T} \\
\mathbf{y}_{\mathbf{3}} & =\left[\begin{array}{ll}
q_{3} & \dot{q}_{3}
\end{array}\right]^{T}
\end{aligned}\right.
$$

where $m_{2}=1.0$ is the mass of mass $2, m_{3}=1.0$ is the mass of mass $3, q_{2}$ is the displacement of mass $2, q_{3}$ is the displacement of mass $3, k_{23}=1.0$, and $\mu_{23}=0.5$. The controllers on component 2 are

$$
\left\{\begin{array}{l}
u_{2}=-\left(\frac{0.1}{s}+0.2 s+0.5\right) q_{2} \\
u_{3}=-(0.6 s+1) q_{3}
\end{array}\right.
$$

The controllers for components 1 and 2 have been designed to produce stable behavior when the components are unconnected. The two components are joined by a spring, $k_{12}=1.0$ connecting mass 1 with mass 2 . The connection parameter, $\epsilon$, multiplies the spring connecting the two components.

A Simulink model of this system has been created to study its stability. The Evolving System was evaluated in its fully evolved state, where the local component controllers were connected to their inputs and outputs and $\epsilon$ was set equal to 1 . Figure 1 shows the nondimensional mass displacements for the Evolving System given by (54)-(56). When the system is fully evolved, i.e., $\epsilon=1$, the Evolved System is unstable as seen in Fig. 1.

A Simulink model was created to implement the adaptive key component controller given by (8) for the illustrative Nonlinear Evolving System example. Simulations were run with the connection parameter, $\epsilon$, ranging from 0 to 1 , allowing analysis of the full evolution of the system. The key component controller was able to maintain system stability during the entire evolution process when it used the input-output ports on mass 1 of component 1, see Fig. 2.

\section{Conclusions}

We provided the reader with the framework for Nonlinear Evolving Systems of flexible structures. We developed the idea of modeling the contact dynamics between components as the feedback connection of the impedance of one component with the admittance of a second component. 
A major contribution made by this paper was the use of the impedance-admittance formulation to deliver theory on the inheritance of dissipativity, passivity, and positive real traits of systems connected in feedback. Using the impedance-admittance formulation of contact dynamics between components of Evolving Systems, we were able to show that strict and almost strict dissipativity and passivity traits are naturally inherited in Nonlinear Evolving Systems. We also showed that for LTI systems, strict dissipativity, strict passivity, and strict positive realness are equivalent properties, with the strict dissipativity trait having the weakest hypotheses. A principal contribution of this paper is the development of the adaptive key component controller and sufficient conditions for its use in Linear and Nonlinear Evolving Systems. Finally, we demonstrated the adaptive key component controller restoring stability in an illustrative example of a Nonlinear Evolving System.

[1] M. J. Balas, S. A. Frost, F. Y. Hadaegh, Evolving systems: A theoretical foundation, in: Proceedings of the AIAA Guidance, Navigation, and Control Conference, Keystone, CO, 2006.

[2] S. A. Frost, Evolving systems: Control and stability inheritance in self-assembling structures, Ph.D. thesis, University of Wyoming, Laramie, WY (2008).

[3] E. D. Flinn, Delicate dance for mango and tango, Aerospace America.

[4] S. A. Frost, M. J. Balas, in: T.T. Arif (Ed.), Aerospace Technologies Advancements, In-Tech Publishing, Austria, 2010, Ch. Evolving Systems and Adaptive Key Component Control.

[5] N. R. Sandell, Jr., M. Athans, P. Varaiya, M. G. Safonov, Survey of decentralized control methods for large scale systems, IEEE Transactions on Automatic Control AC-23 (2) (1978) 108-28.

[6] D. T. Gavel, D. D. Šiljak, Decentralized adaptive control: Structural conditions for stability, IEEE Transactions on Automatic Control 34 (4).

[7] W.-M. Shen, P. Will, B. Khoshnevis, Self-assembly in space via self-reconfigurable robots, Robotics and Automation, 2003. Proceedings. ICRA '03. IEEE International Conference on 2 (2003) 2516-2521 vol.2. doi:10.1109/ROBOT.2003.1241971.

[8] H. Suri, P. Will, W.-M. Shen, System design of robots for application to in-space assembly, in: IEEE International Conference on Robotics and Automation, Beijing, China, 2006.

[9] S. A. Frost, M. J. Balas, Stability inheritance and contact dynamics of flexible structure evolving systems, in: Proceedings 17th IFAC Symposium on Automatic Control in Aerospace, Toulouse, France, 2007.

[10] C. M. Harris, C. E. Crede, Shock and Vibration Handbook, 2nd Edition, McGraw-Hill, New York, NY, 1976.

[11] S. A. Frost, M. J. Balas, Stabilizing controllers for evolving systems with application to flexible space structures, in: Proceedings of the AIAA Guidance, Navigation, and Control Conference, Hilton Head, SC, 2007.

[12] S. A. Frost, M. J. Balas, Adaptive key component controllers for evolving systems, in: AIAA Guidance, Navigation, and Control Conference, Honolulu, HI, 2008

[13] J. Willems, Dissipative dynamical systems part i: General theory, Archive for Rational Mechanics and Analysis 45 (5) (1972) 321-351.

[14] D. Hill, P. Moylan, The stability of nonlinear dissipative systems, IEEE Trans. Automat. Control 21 (5) (1976) 708-711.

[15] W. L. Brogan, Modern Control Theory, 3rd Edition, Prentice-Hall, Englewood Cliffs, NJ, 1991.

[16] M. Vidyasagar, Nonlinear Systems Analysis, 2nd Edition, Prentice-Hall, Englewood Cliffs, NJ, 1993.

[17] M. J. Balas, S. Harvey, E. Mehiel, Nonlinear adaptive control with persistent disturbances, in: Proceedings of the AIAA Guidance, Navigation, and Control Conference, Honolulu, HI, 2008. 


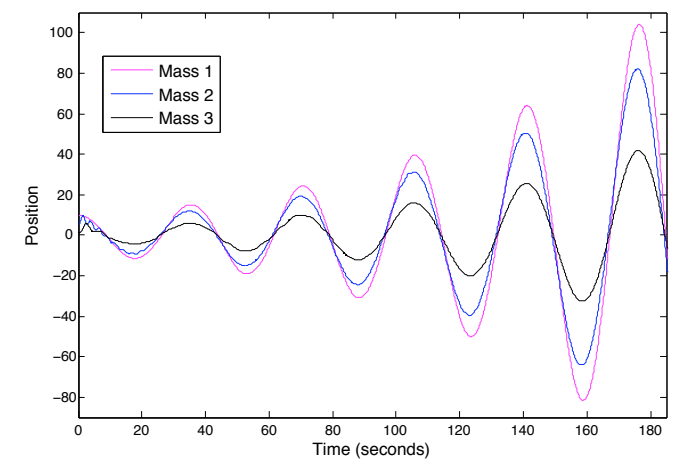

Figure 1: Nondimensional mass displacements of example with no key component control.

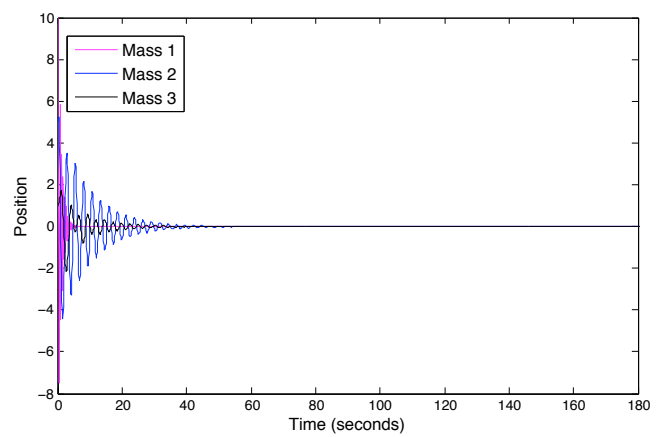

Figure 2: Nondimensional mass displacements of example with adaptive key component controller on component 1. 